\title{
Synchronization and peak-to-peak dynamics in networks of low-dimensional chaotic oscillators
}

Stefano Maggi (maggi@@elet.polimi.it)

Sergio Rinaldi (sergio.rinaldi@polimi.it)

Approved by

Ulf Dieckmann

Program Leader, Evolution and Ecology Program

December 2006 Institute, its National Member Organizations, or other organizations supporting the work. 


\section{Contents}

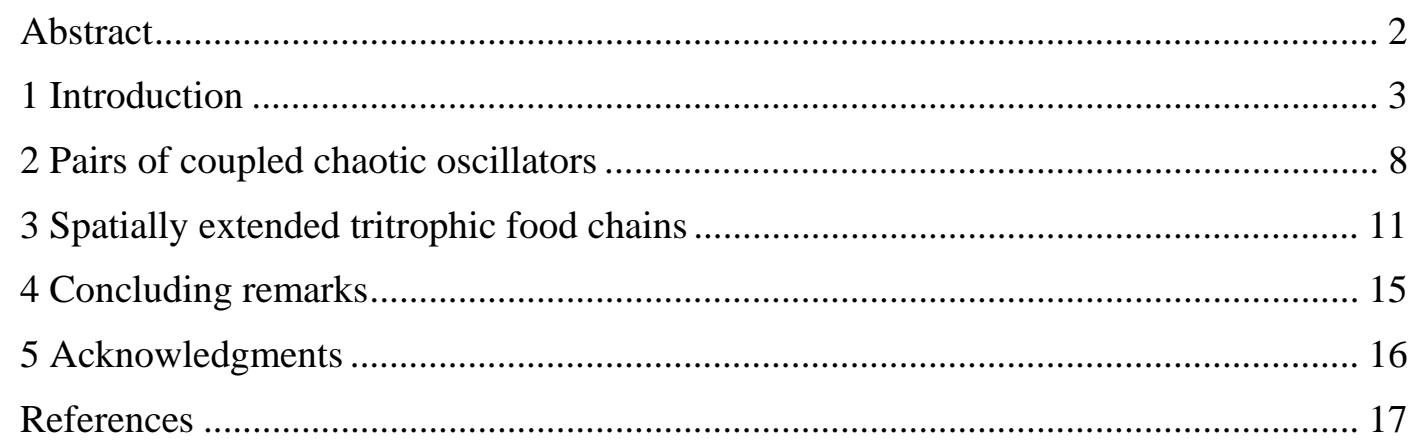




\title{
Synchronization and peak-to-peak dynamics in networks of low-dimensional chaotic oscillators
}

\author{
Stefano Maggi Sergio Rinaldi
}

keywords: networks, chaotic oscillators, synchronization, peak-to-peak dynamics, coupling, coherence

running title: Synchronization and peak-to-peak dynamics

\section{Authors affiliations and e-mails}

Stefano Maggi: Dipartimento di Elettronica e Informazione, Politecnico di Milano, Milano, Italy. E-mail: maggi@elet.polimi.it.

Sergio Rinaldi: Dipartimento di Elettronica e Informazione, Politecnico di Milano, Milano, Italy; Evolution and Ecology Program, International Institute for Applied Systems Analysis, 2361 Laxenburg, Austria. E-mail: rinaldi@elet.polimi.it

\section{Corresponding author}

Sergio Rinaldi, Dipartimento di Elettronica e Informazione, Politecnico di Milano, Via Ponzio 34/5, 20133, Milano, Italy; e-mail rinaldi@elet.polimi.it; phone: +39 022399 3563; fax:+39 0223993412. 


\begin{abstract}
In this paper we study the relationships between local and global properties in networks of dynamical systems by focusing on two global properties, synchronization and peak-to-peak dynamics, and on two local properties, coherence of the components of the network and coupling strength. The analysis is restricted to networks of low-dimensional chaotic oscillators, i.e. oscillators which have peak-to-peak dynamics when they work in isolation. The results are obtained through simulation, first by considering pairs of coupled Lorenz, Rössler and Chua systems, and then by studying the behavior of spatially extended tritrophic food chains described by the Rosenzweig-MacArthur model. The conclusion is that synchronization and peak-to-peak dynamics are different aspects of the same collective behavior, which is easily obtained by enhancing local coupling and coherence. The importance of these findings is briefly discussed within the context of ecological modelling.
\end{abstract}




\section{Introduction}

As is well known, coupling strength controls synchronization in networks of dynamical systems ([Pecora \& Carrol, 1990; Pecora \& Carroll, 1998; Pikovsky et al., 2001; Boccaletti et al., 2002; Manrubia et al., 2004]). In other words, a property concerning local interactions among components of the network turns out to be strategically important for the existence of simple global behaviors. In this paper we extend the analysis of the relationships between local and global phenomena in networks of various topologies by focusing on two global and two local properties. The analysis is restricted to networks of $N$ similar but not identical low-dimensional chaotic oscillators linearly coupled with their nearest neighbors. The fact that the components of the network are not identical precludes any analytical treatment, and in fact our analysis is based on numerical simulations.

The interest for this class of networks is motivated by an ecological application. Indeed, networks of similar but not identical oscillators can be used to model species living in slightly different regions and flowing through the boundaries separating adjacent regions at a rate proportional to the difference of the animal and plant abundances in the two regions ([Okubo, 1980]). Finally, the assumption of low-dimensionality of each chaotic component of the network, which a priori seems rather restrictive, is actually not very limiting in ecology, as shown by a survey of the most common ecological models and of the most studied (laboratory and field) ecological time-series ([Rinaldi et al., 2001; Candaten \& Rinaldi, 2003]).

The two local properties considered in the paper are coupling and coherence. As for the coupling mechanism, we simply assume that if $x^{i}$ is the state vector of the $i$-th oscillator and $S_{i}$ is the set of oscillators coupled with it, the state equation is

$$
\dot{x}^{i}=f\left(x^{i}, p^{i}\right)+\varepsilon D\left(\sum_{j \in S_{i}} x^{j}-m_{i} x^{i}\right)
$$

where $\varepsilon$ is a positive scalar, called coupling strength, $D$ is a specified diagonal matrix with non-negative diagonal entries, $m_{i}$ is the cardinality of $S_{i}$ and $p^{i}$ is 
the parameter vector characterizing the $i$-th component of the network. Notice that eq. (1) could be made more general by assuming that the coupling matrix $D$ depends upon $i$. However, we use here eq. (1) since in all the experiments we have performed we have used constant coupling matrices.

By contrast, coherence is a property concerning each oscillator when it works in isolation $(\varepsilon=0$ in eq. (1)). Highly coherent oscillators are characterized by a small variability of their ups and downs and, indeed, periodic oscillators are infinitely coherent. Among the various ways of defining and measuring coherence we have chosen the one used by [Liu \& Lai, 2001] which is nothing but the $\mu_{i} / \sigma_{i}$ ratio of the return times on a Poincaré section. In particular we use as Poincaré section of each oscillator the manifold on which a scalar variable $s_{i}$ associated to the oscillator, called output variable, is maximum. Although the output variable $s_{i}$ could be any function of the state vector $x^{i}$, in the following it will be identified with one of the components of the vector $x^{i}$, which means that the behavior of each oscillator of the network is observed through one of its state variables. Thus, $\mu_{i}$ and $\sigma_{i}$ are simply the mean and standard deviation of the time intervals separating successive peaks of the output variable $s_{i}$. Of course, both $\mu_{i}$ and $\sigma_{i}$ depend upon the parameter vector $p^{i}$ characterizing the state equation $\dot{x}^{i}=f\left(x^{i}, p^{i}\right)$ of each oscillator, so that it is possible to vary particular components of each vector $p^{i}$ in order to control the coherence of all the oscillators present in the network.

The two global properties discussed in the paper are synchronization and peak-to-peak dynamics. As for synchronization we cannot refer to the extreme notion of complete synchronization, defined as $x^{i}(t) \equiv x^{j}(t) \forall(i, j)$ and $\forall t$ because such a relationship can be satisfied only in networks with identical components. We will therefore consider a weaker form of synchronization, called almost complete synchronization ([Boccaletti et al., 2002]), by looking at the 
correlations between the various oscillators. More precisely, if

$$
R=\left[\begin{array}{cccc}
1 & \rho_{1,2} & \cdots & \rho_{1, N} \\
\rho_{2,1} & 1 & \cdots & \rho_{2, N} \\
\vdots & & \ddots & \vdots \\
\rho_{N, 1} & \rho_{N, 2} & \cdots & 1
\end{array}\right]
$$

is the $N \times N$ correlation matrix, where $\rho_{i, j}=\rho_{j, i}$ is the correlation between the output signals $s_{i}$ and $s_{j}$ when the network is on its attractor, the degree $\rho$ of synchronization is defined as the mean correlation index, i.e.

$$
\rho=\frac{2}{N(N-1)} \sum_{i<j}^{N} \rho_{i, j} .
$$

Obviously, complete synchronization corresponds to $\rho=1$, while values of $\rho$ slightly smaller than 1 correspond (by definition) to almost complete synchronization, and are associated to networks in which all oscillators, observed through their output variables, behave roughly in the same way. In particular, in almost synchronized networks, outputs $s_{i}$ of all oscillators peak almost at the same time (we exclude here exceptional cases like those described in [Balmforth et al., 2000]).

As for peak-to-peak dynamics, let us first recall what they are. For this consider a nonlinear $n$-dimensional continuous time system observed through a scalar output variable $s(t)$ and assume that the system behaves on its attractor. Thus, an infinite series of peaks $\hat{s}^{1}, \hat{s}^{2}, \hat{s}^{3}, \ldots$ can be extracted from the signal $s(t)$ and used to obtain the peak-to-peak plot which is nothing but the set of all pairs $\left(\hat{s}^{k}, \hat{s}^{k+1}\right)$ in $R^{2}$. The peak-to-peak plot is obviously composed of $K$ points if the attractor is a limit cycle and if the output variable peaks $K$ times during the entire cycle. By contrast, if the system is chaotic the peak-topeak plot is a fractal set. Moreover, in the case of low-dimensional chaos, i.e. when the fractal dimension of the strange attractor in $R^{n}$ is close to 2 (as it is in the most known three-dimensional chaotic models ([Candaten \& Rinaldi, 2000])) the fractal dimension of the peak-to-peak plot is close to 1 , so that it can be fairly well approximated by its so-called skeleton. i.e. by one or more curves in $R^{2}$. Very often the skeleton is actually a single curve described by 
a one-dimensional map $\hat{s}^{\prime}=F(\hat{s})$ which allows one to approximately forecast the next peak $\hat{s}^{\prime}$ of the output variable, as soon as the last peak $\hat{s}$ is available. For this reason the map $F$ is called peak-to-peak map and the system is said to have peak-to-peak dynamics, from now on abbreviated as PPD. Thus, in conclusion, in low-dimensional chaotic systems the prediction of the peaks of any output variable does not require $n$ differential equations but much more simply only a one-dimensional map. This is a very important result because in many applications, in particular in those concerning social and environmental sciences, the prediction of the peaks of a specific output variable is often the problem of major concern. Actually, also the time $\tau$ separating two successive peaks can be predicted from the value $\hat{s}$ of the last peak, through a one-dimensional map $\tau=G(\hat{s})$. Therefore, in low-dimensional chaotic systems the dynamics of the peaks is fully described by the following equations

$$
\hat{s}^{\prime}=F(\hat{s}) \quad \hat{t}^{\prime}=\hat{t}+G(\hat{s})
$$

where $\hat{t}$ and $\hat{t}^{\prime}$ are the times of occurrence of two successive output peaks.

Coming back to our problem, let us first notice that the assumption that all chaotic oscillators $\dot{x}^{i}=f\left(x^{i}, p^{i}\right)$ of the network are low-dimensional is equivalent to say that they all have PPD. In other words, each oscillator is endowed of a pair of maps $\left(F_{i}, G_{i}\right)$ that could be used to predict through eqs. (2) the next peak $\hat{s}_{i}^{\prime}$ and its time of occurrence $\hat{t}_{i}^{\prime}$ from the last peak $\hat{s}_{i}$ and its time of occurrence $\hat{t}_{i}$, when the oscillator works in isolation. Notice, however, that the possibility of predicting the future peaks of each uncoupled oscillator does not imply that the peaks of a scalar output variable $s$ associated to the set of all oscillators can be predicted. If, for example, $s$ is the weighted mean of the output variables of the oscillators, i.e. if

$$
s=\sum_{i=1}^{N} \lambda_{i} s_{i}
$$

where $0 \leq \lambda_{i} \leq 1, \sum_{i} \lambda_{i}=1$, the peaks of $s$ occur when $\sum_{i} \lambda_{i} \dot{s}_{i}=0$ and this does not necessarily imply that $\dot{s}_{i}=0 \forall i$ (in the following $\lambda_{i}=1 / N \forall i$ ). Thus, using a continuity argument, we can say that the mismatch of the times of occurrence of the output peaks of the $N$ uncoupled oscillators $(\varepsilon=0$ in (1)) 
prevents the possibility of predicting the peaks of the output variable $s$ of the network when $\varepsilon$ is very small. By contrast, if $\varepsilon$ is increased it might be that some form of synchronization attenuates the mismatch of the times of occurrence of the local peaks so that PPD can emerge at the global scale. In such a case the next peak $\hat{s}^{\prime}$ of the output of the network (see (3)) and its time of occurrence $\hat{t}^{\prime}$ can be predicted from the last peak $\hat{s}$ and from its time of occurrence $\hat{t}$ through eqs. (2) where $F$ and $G$ are one-dimensional maps depending upon all parameters $p^{i}$ characterizing the oscillators and upon the coupling strength $\varepsilon$.

The previous discussion, mainly based on intuitive arguments, brings us naturally to conjecture that synchronization and network PPD are simply different aspects of the same dynamic phenomenon, namely the attenuation of the mismatch in the rhythms at which the components of the network tend to oscillate. It is therefore natural to expect that synchronization and global PPD emerge not only when coupling strength is increased but also when coherence of the oscillators is increased, because higher local coherence means smaller variability in the rhythms locally produced in the network.

In order to support the above conjecture we present a rather systematic study performed on various networks. More precisely, in the next section we study pairs of coupled chaotic oscillators, but for three different types of oscillators, namely Lorenz system ([Lorenz, 1963]), Rössler oscillator ([Rössler, 1976]), and Chua circuit ([Pivka et al., 1996]). Of course the analysis is performed by varying local coherence and coupling strength and by fixing parameter values which guarantee that these oscillators produce low-dimensional chaos when they are uncoupled. Then in the third section we verify that the conjecture holds also in more complex networks. For this we consider a very simple spatially extended ecosystem composed of resource, consumer, and predator and we describe the interactions among the three populations with a third order continuous-time model which exhibits low-dimensional chaotic behavior for suitable values of its parameters. We also assume that the territory where these populations interact is composed of $N$ homogeneous regions characterized by slightly different parameter values and that migration is possible between adjacent regions. In par- 
ticular, we consider three possible territorial topologies which give rise to three different networks: a pair of coupled oscillators $(N=2)$, a one-dimensional closed array of locally interacting oscillators $(N=50)$, and a rectangular array of locally coupled oscillators $(N=20 \times 20)$. Finally, in the last section we

point out open questions and show very briefly the potential of our findings by commenting on the studies concerning the longest chaotic ecological time series, namely that of the Canadian Lynx ([Elton \& Nicholson, 1942]).

\section{Pairs of coupled chaotic oscillators}

This section discusses the simplest networks of chaotic oscillators, namely those composed of only two coupled oscillators. Three prototypes, namely Lorenz, Rössler and Chua systems, are used for the experiments. We show that almost complete synchronization is needed to achieve network PPD. Moreover, our analysis points out that lower coupling strength is needed to achieve synchronization when the oscillators have higher coherence.

The section is structured as follows. First we recall the state equations of the three oscillators and check that for the proposed parameter values the oscillators have low-dimensional chaos no matter if their coherence is high or low. Then, we specify the coupling mechanism (i.e. the matrix $D$ in (1)) and report the results obtained at the network scale in terms of synchronization and PPD for various values of the coupling strength and of the local degree of coherence.

\section{The Lorenz oscillator}

The Lorenz system is described by the following state equations ([Lorenz, 1963])

$$
\begin{aligned}
& \dot{x_{1}}=\sigma_{1}\left(x_{2}-x_{1}\right) \\
& \dot{x_{2}}=r x_{1}-x_{2}-x_{1} x_{3} \\
& \dot{x_{3}}=x_{1} x_{2}-b_{1} x_{3}
\end{aligned}
$$

and the associated output variable is the third state variable. Reference parameter values are $\sigma=10, b=8 / 3$, while $r \in[33,52]$ is used to control coherence, 
which decreases with respect to $r$. For these parameter values the Lorenz oscillator has PPD as shown in the upper block (first two rows) of Fig. 1. In the first and second column of this figure the maps $F$ and $G$ used in (2) are shown.

The Lorenz system is not very coherent; in fact, the range of its return times is rather large as shown in the second column of Fig. 1. In particular, both the high and low coherence regimes present a pronounced tail toward high return times, caused by the slowing down that trajectories experience when they pass close to the origin.

\section{The Rössler oscillator}

The state equations of the Rössler oscillator are ([Rössler, 1976])

$$
\begin{aligned}
& \dot{x_{1}}=-x_{2}-x_{3} \\
& \dot{x_{2}}=x_{1}+a x_{2} \\
& \dot{x_{3}}=b+x_{1} x_{3}-c x_{3}
\end{aligned}
$$

and the state variable selected as output is the second one. Reference parameter values are $a=0.2, b=0.2$, while parameter $c \in[5,9]$ controls coherence which decreases with respect to $c$. The peak-to-peak plots from which the maps $F$ and $G$ can be derived are shown in the second block of Fig. 1. As shown in the second column of Fig. 1 the Rössler system, yet being chaotic, can be quite coherent or poorly coherent, depending on the value of $c$. The low coherence regime is often called "funnel regime" ([Blasius \& Stone, 2000; Pikovsky et al., 2001]) because of the tight swirls its trajectories produce when projected on the $\left(x_{1}, x_{2}\right)$ plane. 


\section{The Chua oscillator}

The Chua circuit is described by the following state equations ([Pivka et al., 1996]):

$$
\begin{aligned}
& \dot{x_{1}}=a\left(x_{2}-b x_{1}^{3}-c x_{1}\right) \\
& \dot{x_{2}}=x_{1}-x_{2}+x_{3} \\
& \dot{x_{3}}=-d x_{2}-e x_{3} .
\end{aligned}
$$

and the state variable considered as output is the second one. Reference parameter values are $b=-0.5, c=0.2, d=31.25, e=-3.125$, while parameter $a \in[-100,-65]$ controls coherence, which increases with it. The peak-to-peak plots for which the maps $F$ and $G$ can be identified are reported in the third block of Fig. 1. Depending on the value of $a$, the Chua system can be very coherent or poorly coherent as shown in the second column of Fig. 1.

We now show the results obtained by simulating pairs of coupled oscillators for each type reported above. As shown in Table 1, the parameter values of the two coupled oscillators are very similar but not identical (small variations with respect to reference values). By contrast, the parameter used to control coherence has been systematically given the same value in both oscillators. As for the coupling matrix $D$, we have made the simplest choice, namely the identity matrix.

The simulations of the network have been performed by integrating eq. (1) with the 4th order Runge-Kutta-Fehlberg method with 5th order error estimate and the degree of synchronization has been measured with the mean correlation index $\rho$. The results obtained by varying the coupling strength and the parameter controlling coherence in relatively large ranges are shown in Fig. 2 which points out that almost complete synchronization is obtained for high values of coupling and coherence. It is easy to notice that oscillators with higher coherence need less coupling strength to synchronize. For example, in the case of the Chua circuit, in the high coherence regime $(a=-65)$ almost complete synchro- 
nization emerges already at the relatively weak coupling strength of $\varepsilon=0.075$, while in the low coherence regime $(a=-100)$ almost complete synchronization cannot be achieved even with a three times higher coupling strength.

As for global PPD we could produce a similar figure if we would be able to define a synthetic indicator telling us if the network has or not (at least approximately) PPD. The most natural indicator is the fractal dimension of the peak-to-peak plot associated to the output $s=\frac{1}{2}\left(s_{1}+s_{2}\right)$ of the network. However, the few trials we made in this direction did not give sharp results. By contrast, a much more transparent message is obtained by showing explicitly the peak-to-peak plots for a series of combinations of the coupling strength and of the parameter controlling coherence. The result is shown in Fig. 3 where 16 peak-to-peak plots are reported for each type of network. If the coupling strength is very small the two oscillators are not synchronized and the network peak-to-peak plot is a cloud in a two-dimensional space. In other words, if the two oscillators composing the network are very weakly coupled, peak-to-peak dynamics is lost. By contrast, if the coupling strength is increased, PPD can emerge, in particular if the oscillators are coherent.

Moreover, by comparing Figs. 2 and 3 one can conclude that all peak-topeak plots corresponding to values of the parameters which guarantee almost complete synchronization are very narrow clouds around a skeleton, suggesting a one-dimensional peak-to-peak map.

\section{$3 \quad$ Spatially extended tritrophic food chains}

In order to further support our conjecture on the role of local features in the emergence of global phenomena in networks of low-dimensional chaotic oscillators, we present in this section a study on a simple spatially extended ecosystem. For this we consider three populations, resource $\left(x_{1}\right)$, consumer $\left(x_{2}\right)$ and predator $\left(x_{3}\right)$, and use the most standard tritrophic food chain model, namely the so-called Rosenzweig-MacArthur model ([Rosenzweig \& MacArthur, 1963]) to describe the interactions among the three populations in each homogeneous re- 
gion of a given territory. We consider, in particular, three possible cases. In the first one the territory is composed of two regions with possible migrations in both directions (e.g. a forest with a river flowing through it). In the second case the territory is a circular ring composed of various homogeneous regions where migration is possible in two directions (e.g. an ecosystem functioning in a narrow circular band of land, like the border of a lake or of an island). Finally, in the third case the territory is a two-dimensional compact area partitioned in various regions (e.g. a large island with various watersheds). In practice, in our idealized study we will consider three topologically different networks: a pair of coupled oscillators $(N=2)$, a one-dimensional closed array of oscillators $(N=50)$, and a two-dimensional array with reflecting boundary conditions $(N=20 \times 20)$. The Rosenzweig-MacArthur model is

$$
\begin{aligned}
& \dot{x}_{1}=r x_{1}\left(1-\frac{x_{1}}{K}\right)-\frac{a_{2} x_{1}}{b_{2}+x_{1}} x_{2} \\
& \dot{x}_{2}=e_{2} \frac{a_{2} x_{1}}{b_{2}+x_{1}} x_{2}-d_{2} x_{2}-\frac{a_{3} x_{2}}{b_{3}+x_{2}} x_{3} \\
& \dot{x}_{3}=e_{3} \frac{a_{3} x_{2}}{b_{3}+x_{2}} x_{3}-d_{3} x_{3}
\end{aligned}
$$

where $r$ and $K$ are the net growth rate and carrying capacity of the resource and $a_{2}, b_{2}, e_{2}, d_{2}\left[a_{3}, b_{3}, e_{3}, d_{3}\right]$ are maximum predation rate, half saturation constant, efficiency, and death rate of consumer [predator]. The model can have chaotic behavior ([Hastings \& Powell, 1991; Kuznetsov \& Rinaldi, 1996; Kuznetsov et al., 2001]). Moreover, the strange attractor is low-dimensional, so that there are peak-to-peak dynamics (as shown below).

The reference parameter values for consumer and predator populations (see (4)) are reported in Table 2. They will be assigned to all oscillators composing the network, while the parameters $r$ and $K$ qualifying the resource will slightly depend upon the position of the oscillator in the network. This means that in our ecosystem only the resource dynamics are not spatially homogeneous. In the following, we focus our attention on the dynamics of the predator, i.e. we select $x_{3}$ as output variable of each oscillator. Figure 4 reports an example of timeseries and shows that the third state variable presents two substantially different kind of peaks: super-peaks, marked with empty circles (and defined as 
peaks dominating the previous and subsequent peaks) and other peaks. Here we only consider super-peaks, for two reasons: first, because they are the most interesting extreme events in the dynamics of a food chain, and second, because each super-peak clearly identifies the end of an oscillation. Figure 5 shows the (super) peak-to-(super) peak plots $\left(\hat{s}, \hat{s}^{\prime}\right)$ (first column) and $(\hat{s}, \tau)$ (second column) obtained for two different values of the parameter $e_{2}$ controlling coherence. In the first row $\left(e_{2}=0.96\right)$ the oscillator is quite coherent $(\mu / \sigma=8.76)$ while in the second row $\left(e_{2}=1.02\right)$ the oscillator is less coherent $(\mu / \sigma=3.75)$. The plots in Fig. 5 are fractal sets which, however, can hardly be distinguished from their skeletons. This proves that the oscillator (4) has low-dimensional chaos for the selected parameter values.

Let us now specify the characteristics of the three networks we have studied. As for the coupling mechanism, we have assigned strongly different values to the three diagonal elements $\left(D_{1}, D_{2}\right.$, and $\left.D_{3}\right)$ of the coupling matrix $D$, because such elements measure the tendency to disperse of the three populations. For example, in the case of a (plant, herbivore, carnivore) food chain, consumer (herbivore) and predator (carnivore) can disperse much more than resource (plant). For this reason, we will first present the results obtained with $D_{3}=$ $D_{2}=100 D_{1}$ and then show the effect of the diversification of $D_{2}$ and $D_{3}$. As for the network topology we consider the following three cases

- pair of coupled oscillators $(N=2)$

- one-dimensional closed array $(N=50)$

- two dimensional array with fully reflecting boundary conditions $(N=$ $20 \times 20)$

For the first [second] network, $m_{i}$ is equal to 1 [2] for all $i$ in eq. (1), while for the third network $m_{i}$ is equal to 2 (at the four corners of the grid), 3 (on the edges of the grid) or 4 (at the interior of the grid).

As already said, all oscillators in the networks have been diversified by assigning slightly different values to the resource growth rate $r$ and the carrying capacity $K$. More precisely, for the pair of coupled oscillators $(r=1.15$, 
$K=1.07)$ in the first oscillator and $(r=1.2679, K=1.05)$ in the second, while the values of $r$ and $K$ in the two other networks are shown in Fig. 6 and mimic a possible spatial dependence due to factors like altitude, exposure to light and wind, humidity, ...

The results concerning the synchronization of the three networks are reported in Fig. 7. As in the previous section, synchronization increases with coupling strength and coherence. Sixteen samples of the peak-to-peak plot of each network are shown in Fig. 8. The figure is very similar to Fig. 3 and shows that the peak-to-peak plots are dispersed clouds of points for low coupling strength and coherence and gradually become one-dimensional curves when coupling strength and coherence are increased. Moreover, the comparison of Figs. 7 and 8 shows, once more, that synchronization and global peak-to-peak dynamics are different aspects of the same phenomenon.

The results we have illustrated are robust. Indeed, fully similar diagrams are obtained when the parameters are modified. Particularly interesting is the analysis of the case of asymmetric dispersion of consumer and predator $\left(D_{2} \neq D_{3}\right)$ because the corresponding synchronization diagram, reported in Fig. 9, points out an interesting result, namely that predator dispersion favors synchronization (and hence PPD) much more than consumer dispersion.

The qualitative results we have pointed out with the Rosenzweig-MacArthur model can be obtained also with other tritrophic food chain models, like those studied in [Candaten \& Rinaldi, 2003] which have low-dimensional chaos. This is true, for example, for the tritrophic food chain model used by Blasius and Stone in their studies on phase synchronization ([Blasius et al., 1999; Blasius \& Stone, 2000]). It is important to notice, however, that phase synchronization is a weak form of synchronization which does not guarantee a strong attenuation of the mismatch of the times of occurrence of the peaks of the oscillators composing the network. For this reason, we have not even considered it in this paper, since phase synchronization is sometimes too weak to generate global peak-to-peak dynamics. 


\section{Concluding remarks}

We have shown in this paper that almost complete synchronization and peakto-peak dynamics are different aspects of the same phenomenon in networks of low-dimensional chaotic oscillators. This implies that one can use the mean correlation index not only for checking if a network is synchronized or not, but also for discovering if it has PPD. Conversely, a simple peak-to-peak plot can be used to point out synchronization.

The key feature for the emergence of synchronization and peak-to-peak dynamics is the attenuation due to local coupling of the mismatch existing among the rhythms at which the components of the network oscillate. Moreover, coherence of the oscillators acts as a catalyst for the emergence of these global phenomena since networks of oscillators with higher coherence systematically synchronize with lower coupling strength.

Due to the interest in a specific application, we have considered networks composed of locally coupled not identical oscillators. This has ruled out the possibility of any analytical treatment, and in fact the results have been derived through extensive simulation. It might be interesting, however, to give a more formal support to our findings by studying the special case of identical oscillators through the Pecora and Carrol master stability equation ([Pecora \& Carroll, 1998]). Another interesting problem is to find out if the results hold also for other classes of networks, obtained by relaxing the assumption of local coupling. Indeed, intuition and analogy with other studies suggest, for example, that the addition of some degree of intermittent long-distance coupling might be another strong catalyst for the emergence of synchronization and peak-to-peak dynamics. Finally, a more important but possibly more difficult extension would be to relax the assumption of low-dimensional chaos of the oscillators composing the network.

However, despite obvious limitations, our findings are of definite importance in the context of ecological modelling. Let us explain this by making reference to a problem that has attracted a great number of scholars, namely the description of the oscillations of various species in various regions of Northern Canada 
(see, for example, [Elton \& Nicholson, 1942]). Over such a large territory the ecosystem is composed of a huge number of interacting populations which can however be roughly considered as a tritrophic food chain where the prototypical herbivore and carnivore are, respectively, the hare and the lynx ([King \& Schaffer, 2001]). Thus, on the basis of the results obtained in the previous section (see Fig. 9) we should not be surprised to be able to detect peak-to-peak dynamics in the lynx numbers of Northern Canada because the lynx can diffuse much more than the hare. A second and more convincing argument in favor of this idea comes from the data collected by Elton and Nicholson ([Elton \& Nicholson, 1942]) on the yearly lynx catches in ten regions of Northern Canada for about one century. Looking at these data, reported in Fig. 10, one can immediately recognize, even without any explicit computation, that the lynx numbers (proportional to the catches) are quite synchronized. This simple observation, together with the results pointed out in this paper, definitely suggests that one should licitly expect peak-to-peak dynamics in the lynx numbers over all Northern Canada. Indeed, some evidence of this astonishingly simple result was recently produced ([Rinaldi et al., 2001]) through the analysis of the data of the MacKenzie River region ( $\lambda_{i}=1$ in (3) for that region). However, the fact that this result is consistent with the conceptual framework pointed out in this paper, makes it more credible.

\section{Acknowledgments}

The authors are grateful to Stefano Boccaletti for his helpful suggestions. Financial support was provided by MIUR under project FIRB2001-RBNE01CW3M. 


\section{References}

Balmforth, N., Pasquero, C. \& Provenzale, A. [2000] "The Lorenz-Fermi-PastaUlam experiment," Physica D 138, 1-43.

Blasius, B., Huppert, L. \& Stone, L. [1999] "Complex dynamics and phase synchronization in spatially extended ecological systems," Nature 399, 354359.

Blasius, B. \& Stone, L. [2000] "Chaos and phase synchronization in ecological systems," International Journal of Bifurcation and Chaos 10, 2361-2380.

Boccaletti, S., Kurths, J., Osipov, G., Valladares, D. L. \& Zhou, C. S. [2002] "The synchronization of chaotic systems," Physics Reports 366, 1-101.

Candaten, M. \& Rinaldi, S. [2000] "Peak-to-peak dynamics: a critical survey," International Journal of Bifurcation and Chaos 10, 1805-1819.

Candaten, M. \& Rinaldi, S. [2003] "Peak-to-peak dynamics in food chain models," Theoretical Population Biology 63, 257-267.

Elton, C. \& Nicholson, M. [1942] "The ten-year cycle in numbers of the lynx in Canada," Journal of Animal Ecology 11, 215-244.

Hastings, A. \& Powell, T. [1991] "Chaos in a three-species food chain," Ecology 72, 896-903.

King, A. A. \& Schaffer, W. M. [2001] "The geometry of a population cycle: A mechanistic model of the snowshoe hare cycle," Ecology 82, 814-830.

Kuznetsov, Y. A., Feo, O. D. \& Rinaldi, S. [2001] "Belyakov homoclinic bifurcations in a tritrophic food chain model," SIAM Journal on Applied Mathematics 62, 462-487.

Kuznetsov, Y. A. \& Rinaldi, S. [1996] "Remarks on food chain dynamics," Mathematical Biosciences 134, 1-33.

Liu, Z. \& Lai, Y.-C. [2001] "Coherence resonance in coupled chaotic oscillators," Physical Review Letters 86(21), 4737-4740. 
Lorenz, E. N. [1963] "Deterministic nonperiodic flow," Journal of the Atmospheric Sciences 20, 130-141.

Manrubia, S. C., Mikhailov, A. S. \& Zanette, D. H. [2004] Emergence of Dynamical Order - Synchronization Phenomena in Complex systems (World Scientific).

Okubo, A. [1980] Diffusion and Ecological Problems: Mathematical Models (Springer-Verlag).

Pecora, L. M. \& Carrol, T. L. [1990] "Synchronization in chaotic systems," Physical Review Letters 64(8), 821-824.

Pecora, L. M. \& Carroll, T. L. [1998] "Master stability functions for synchronized coupled systems," Physical Review Letters 80(10), 2109-2112.

Pikovsky, A., Rosenblum, M. \& Kurths, J. [2001] Synchronization - A Universal Concept in Nonlinear Sciences (Cambridge University Press).

Pivka, L., Wu, C. W. \& Huang, A. [1996] "Lorenz equation and Chua's equations," International Journal of Bifurcation and Chaos 6, 2443-2489.

Rinaldi, S., Candaten, M. \& Casagrandi, R. [2001] "Evidence of peak-to-peak dynamics in ecology," Ecology Letters 4, 610-617.

Rosenzweig, M. L. \& MacArthur, R. H. [1963] "Graphical representation and stability conditions of predator-prey interactions," American Naturalist 97, 209-223.

Rössler, O. E. [1976] "An equation for continuous chaos," Physics Letters A 57, 397-398. 


\section{Table captions}

Tab. 1 Parameter values used in the simulations of pairs of coupled chaotic oscillators.

Tab. 2 Reference parameter values of consumer and predator populations in Rosenzweig-MacArthur model (4). 


\begin{tabular}{|c|c|c|}
\hline & oscillator 1 & oscillator 2 \\
\hline \multirow{2}{*}{ Lorenz } & $\sigma=10$ & $\sigma=10.1$ \\
\hline & $b=8 / 3$ & $b=8 / 3$ \\
\hline \multirow{2}{*}{ Rössler } & $a=0.2$ & $a=0.24$ \\
\hline & $b=0.2$ & $b=0.35$ \\
\hline \multirow{4}{*}{ Chua } & $b=-0.5$ & $b=-0.6$ \\
\hline & $c=0.2$ & $c=0.2$ \\
\hline & $d=31.25$ & $d=31.25$ \\
\hline & $e=-3.125$ & $e=-3.125$ \\
\hline
\end{tabular}


Table 2: Maggi \& Rinaldi 2005

\begin{tabular}{cc|cc}
\multicolumn{2}{c}{ consumer } & \multicolumn{2}{c}{ predator } \\
\hline$a_{2}$ & $5 / 3$ & $a_{3}$ & $1 / 20$ \\
$b_{2}$ & $1 / 3$ & $b_{3}$ & $1 / 2$ \\
$e_{2}$ & 1 & $e_{3}$ & 1 \\
$d_{2}$ & $4 / 10$ & $d_{3}$ & $1 / 100$
\end{tabular}




\section{Figure captions}

Fig. 1 Peak-to-peak plots $\left(\hat{s}, \hat{s}^{\prime}\right)$ (first column) and $(\hat{s}, \tau)$ (second column) for the three kinds of oscillators. Parameter values are at their reference values (indicated in the text). In each block the first [second] row reports the case of highest [lowest] coherence $\mu / \sigma$. The skeletons of all these peakto-peak plots (which are fractal sets) are the peak-to-peak maps denoted by $F$ (first column) and $G$ (second column) in the text (see eq. (2)).

Fig. 2 The degree of synchronization $\rho$ in the three types of networks as a function of coupling strength $\varepsilon$ and of the parameter controlling coherence. In each diagram coherence increases going upward. Parameter values of the oscillators composing each network are in Table 1.

Fig. 3 Sixteen peak-to-peak plots $\left(\hat{s}, \hat{s}^{\prime}\right)$ for each one of the three types of networks considered in the text. Each plot has been obtained for the parameter values specified in Table 1 and for the values of the coupling strength and of the parameter controlling coherence specified on the axis. In each diagram coherence increases going upward.

Fig. 4 Timeseries of the third state variable (predator) in the RosenzweigMacArthur model (4). Empty circles identify the so-called super-peaks. Parameters as in Table 2, $r=1.15, K=1.07$.

Fig. 5 Peak-to-peak plots $\left(\hat{s}, \hat{s}^{\prime}\right)$ (first column) and $(\hat{s}, \tau)$ (second column) for the Rosenzweig-MacArthur model (4). The first row corresponds to the most coherent regime $\left(e_{2}=0.96, \mu / \sigma=8.76\right)$ while the second row corresponds to the less coherent regime $\left(e_{2}=1.02, \mu / \sigma=3.75\right)$. All other parameters are as in Table 2, $r=1.15, K=1.07$.

Fig. 6 Dependence of $r$ and $K$ upon the position of the oscillator in the network: (a) one dimensional closed array with $N=50$; (b) and (c) twodimensional array with $N=20 \times 20$.

Fig. 7 The degree of synchronization $\rho$ in the three networks composed of 
Rosenzweig-MacArthur oscillators. In each diagram coherence increases going upward. Parameter values as in Table $2, r$ and $K$ as in Fig. 6.

Fig. 8 Sixteen peak-to-peak plots $\left(\hat{s}, \hat{s}^{\prime}\right)$ for each one of the three types of networks composed of Rosenzweig-MacArthur oscillators. Each plot has been obtained for the parameter values specified in Table 1 and for the values of the coupling strength and of the parameter controlling coherence specified on the axis. In each diagram coherence increases going upward.

Fig. 9 The degree of synchronization $\rho$ in a two-dimensional network of RosenzweigMacArthur oscillators with asymmetric dispersion of consumer and predator.

Fig. 10 Lynx fur returns in 5 regions of Northern Canada (reproduced from [Elton \& Nicholson, 1942]). 

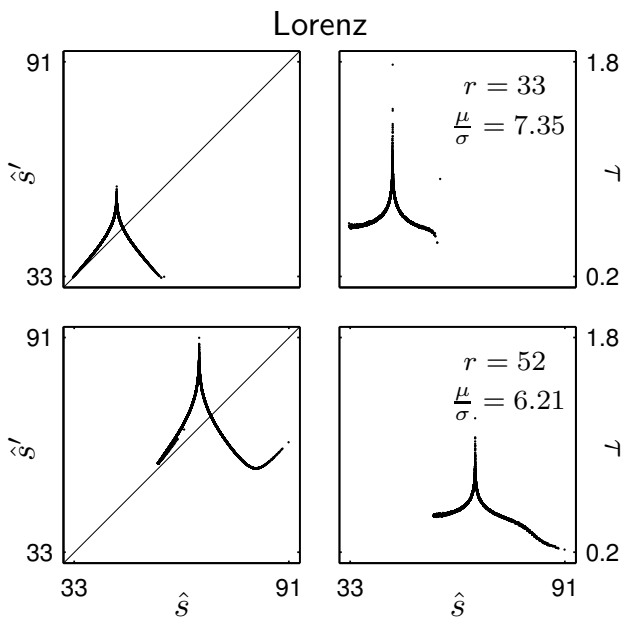

Röessler
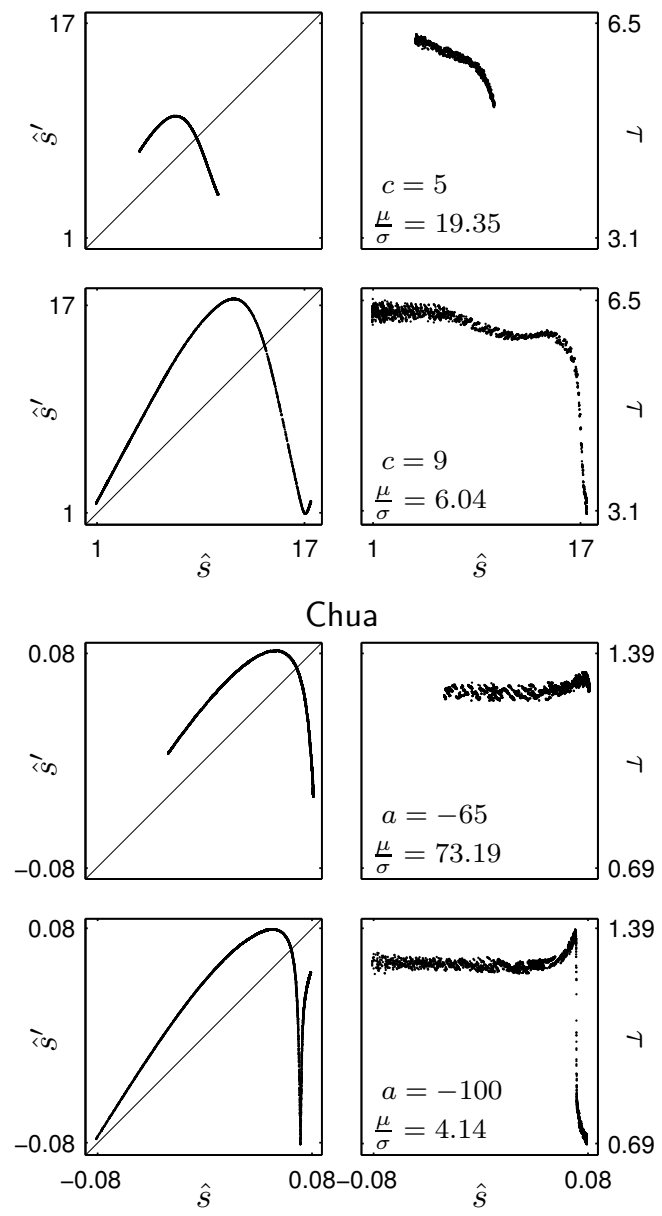

Figure 1: Maggi \& Rinaldi 2005 

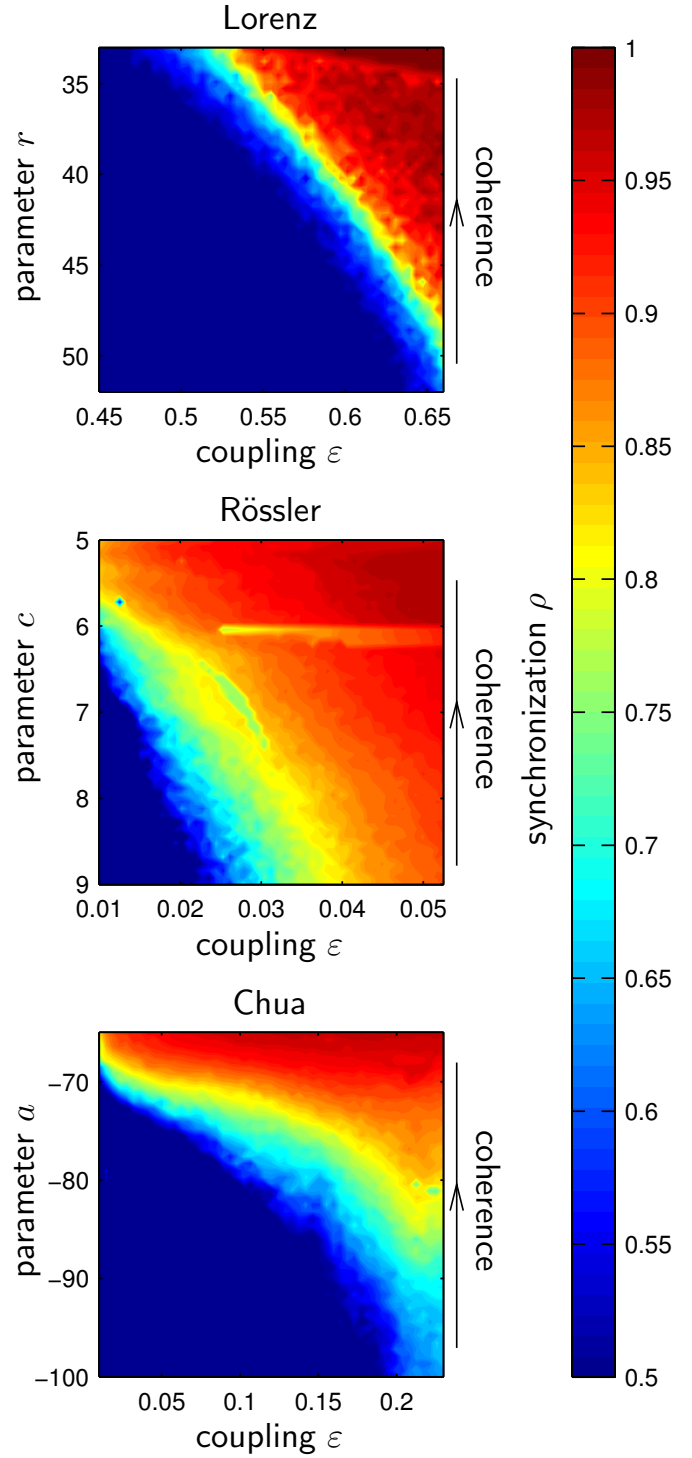

Figure 2: Maggi \& Rinaldi 2005 

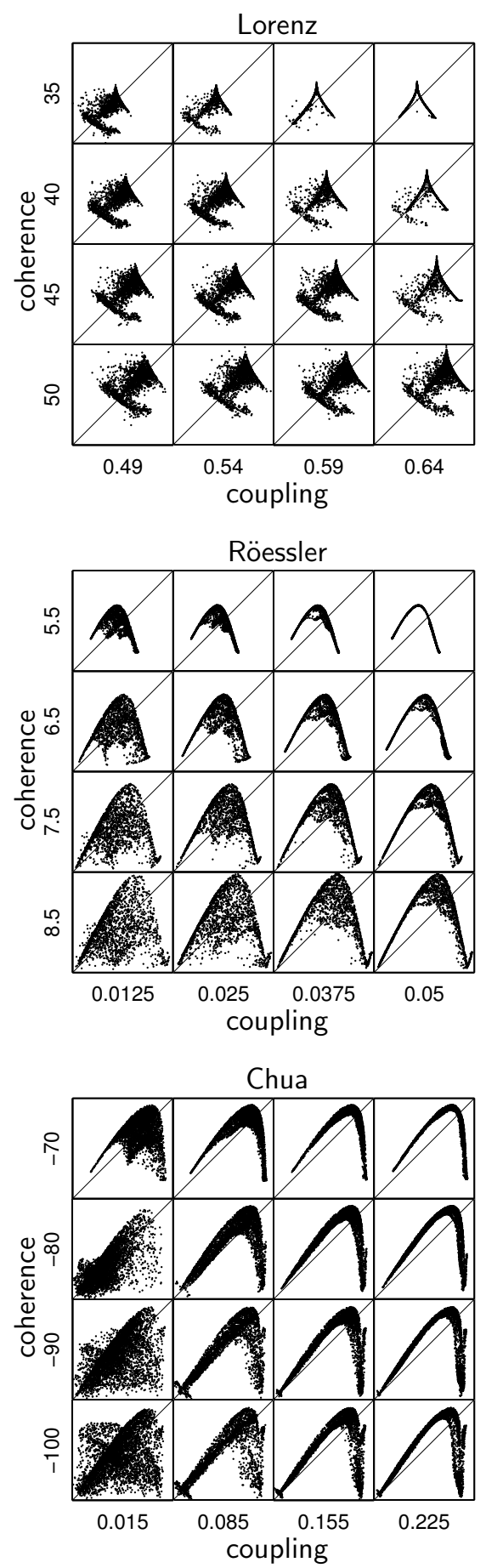

Figure 3: Maggi \& Rinaldi 2005 


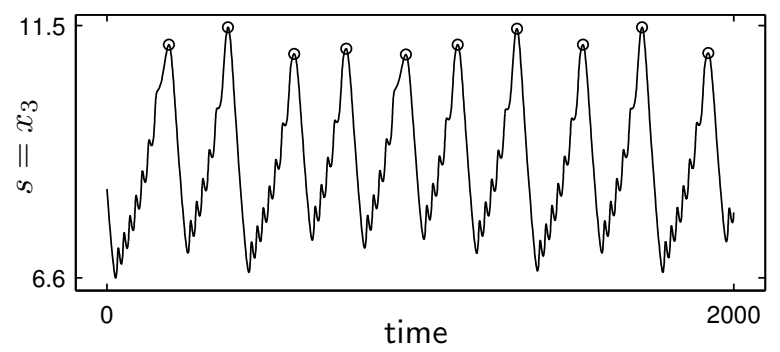

Figure 4: Maggi \& Rinaldi 2005 

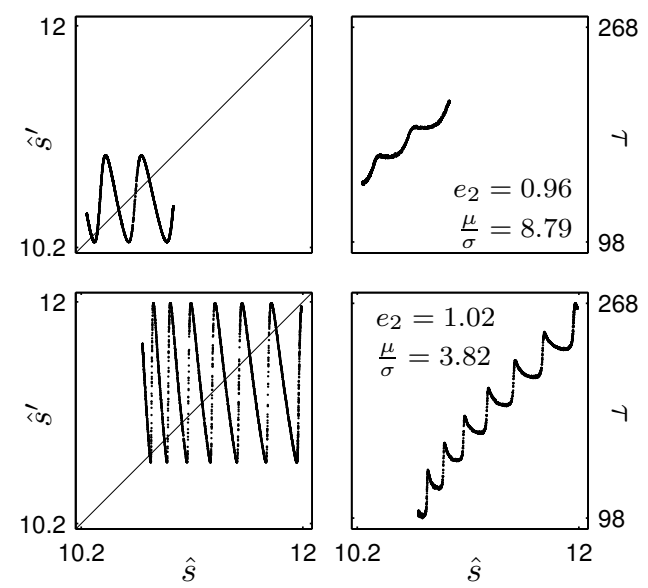

Figure 5: Maggi \& Rinaldi 2005 

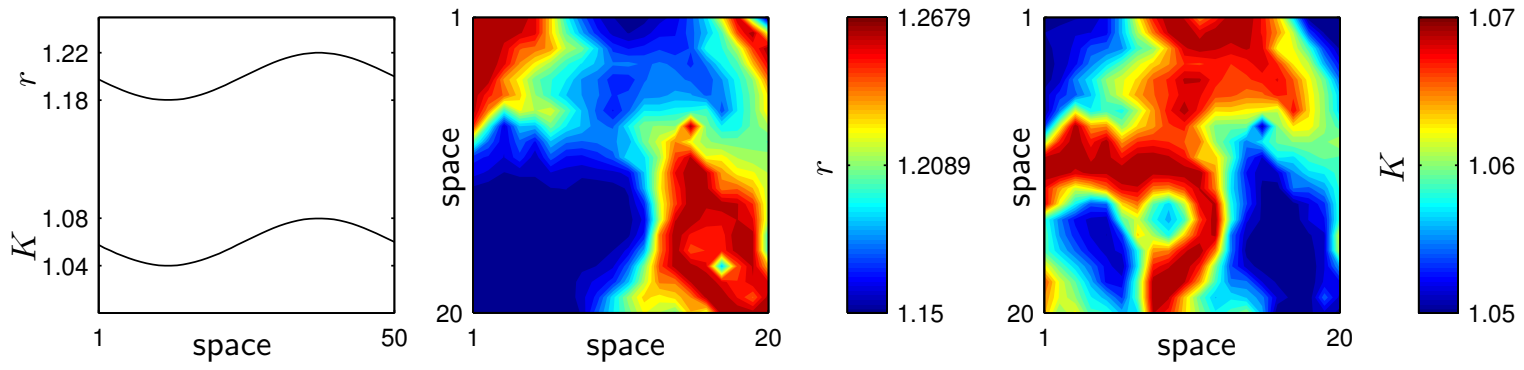

Figure 6: Maggi \& Rinaldi 2005 

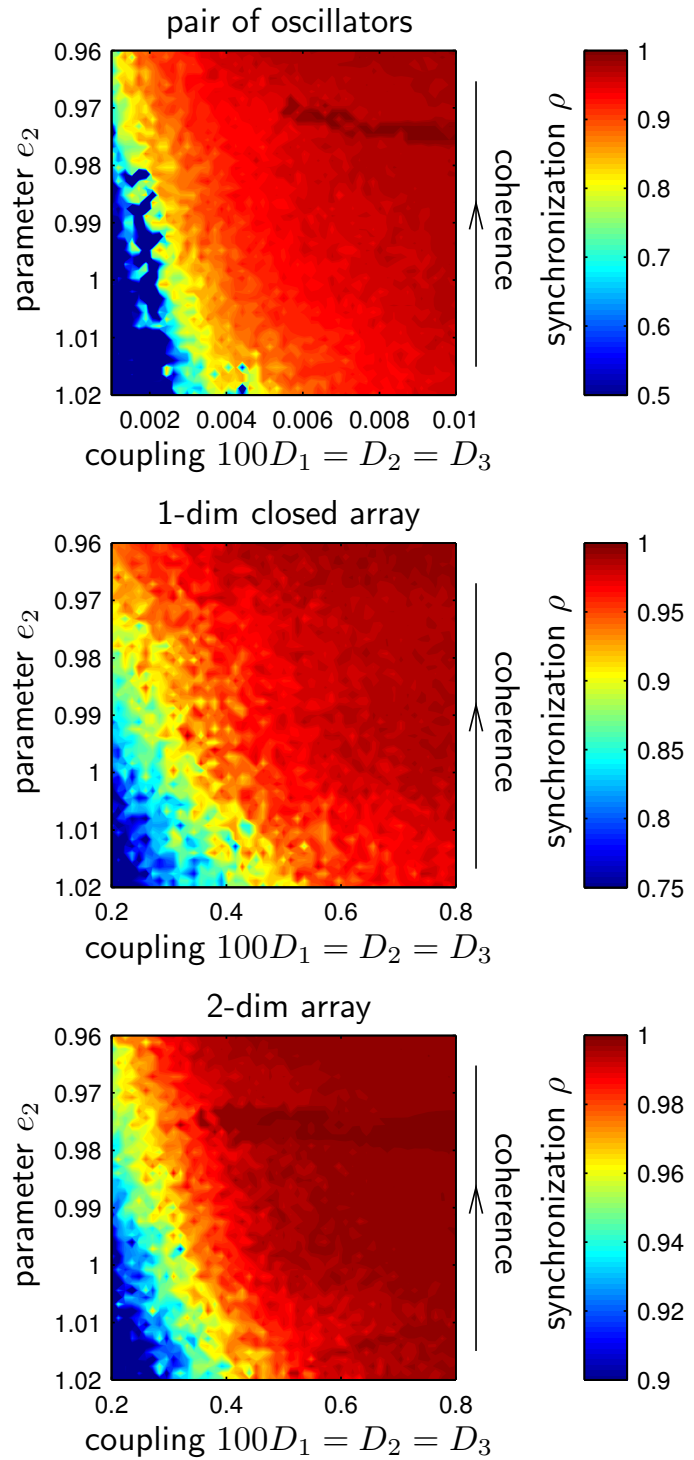

Figure 7: Maggi \& Rinaldi 2005 

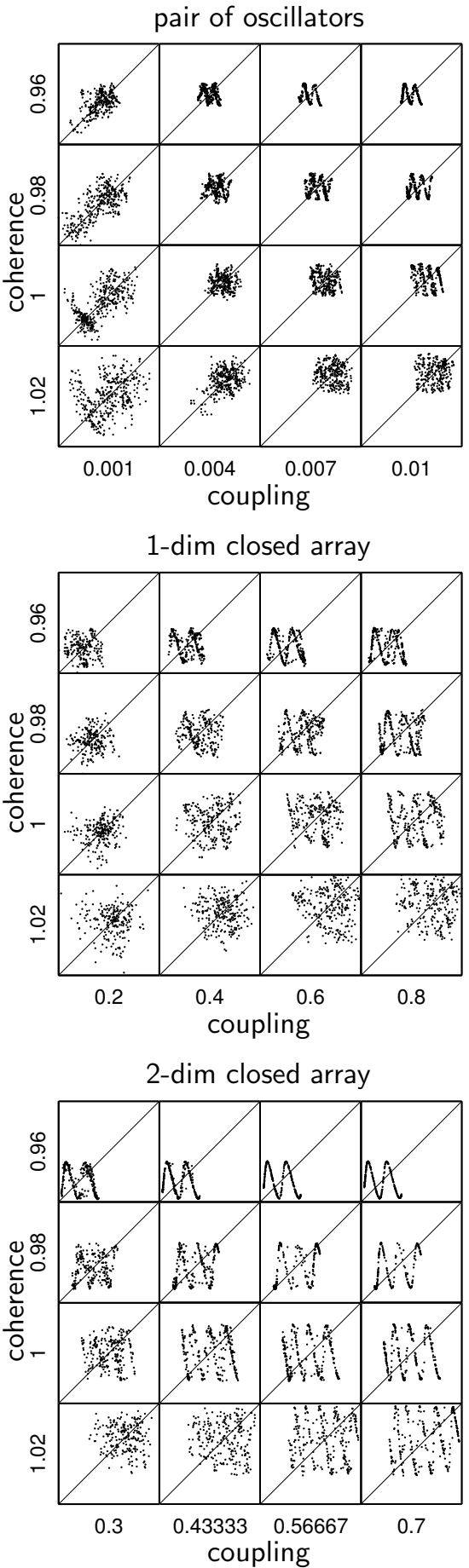

Figure 8: Maggi \& Rinaldi 2005 


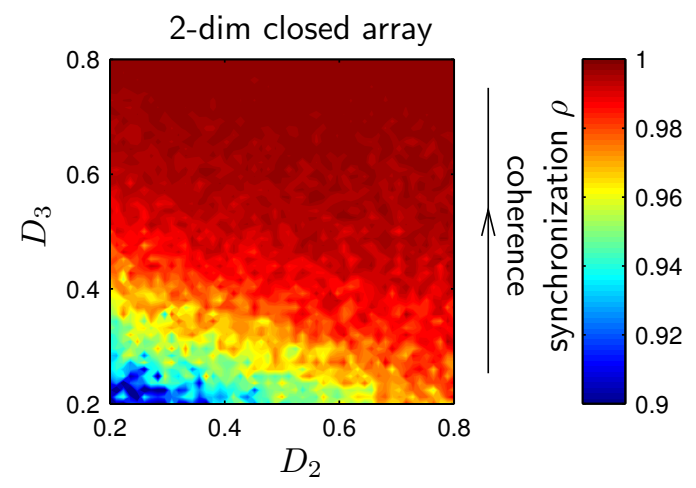

Figure 9: Maggi \& Rinaldi 2005 


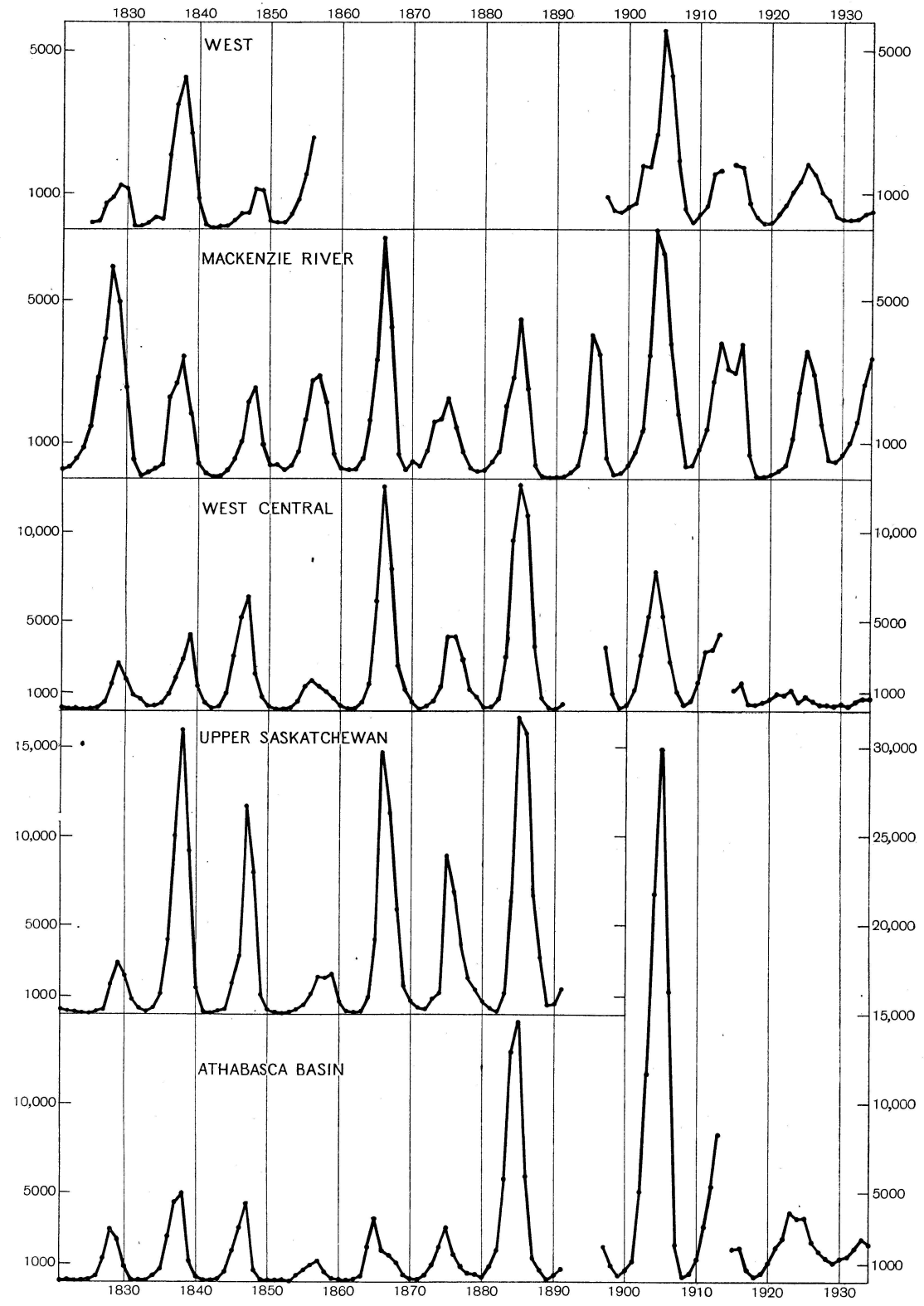

Figure 10: Maggi \& Rinaldi 2005 